\title{
CryptRndTest: An R Package for Testing the Cryptographic Randomness
}

by Haydar Demirhan and Nihan Bitirim

\begin{abstract}
In this article, we introduce the R package CryptRndTest that performs eight statistical randomness tests on cryptographic random number sequences. The purpose of the package is to provide software implementing recently proposed cryptographic randomness tests utilizing goodnessof-fit tests superior to the usual chi-square test in terms of statistical performance. Most of the tests included in package CryptRndTest are not available in other software packages such as the R package RDieHarder or the C library TestU01. Chi-square, Anderson-Darling, Kolmogorov-Smirnov, and Jarque-Bera goodness-of-fit procedures are provided along with cryptographic randomness tests. CryptRndTest utilizes multiple precision floating numbers for sequences longer than 64-bit based on the package Rmpfr. By this way, included tests are applied precisely for higher bit-lengths. In addition CryptRndTest provides a user friendly interface to these cryptographic randomness tests. As an illustrative application, CryptRndTest is used to test available random number generators in $\mathrm{R}$.
\end{abstract}

\section{Introduction}

Cryptographic random numbers constitute the heart of ciphering processes. Security of the transmitted information is mostly based on the quality of random numbers used to cipher the information. Due to efficiency considerations, pseudo random numbers that ensure some hard-to-achieve properties are used for ciphering in practice. There are a considerable amount of pseudo random number generators (RNG's) in the literature of cryptography. Suitability of these RNG's for use in cryptographic applications is evaluated based on statistical randomness tests that are specifically designed to test randomness at the level required for ciphering processes.

In a cryptographic randomness test, first, the empirical distribution of a test statistic is obtained over a random number sequence by various data manipulations. Then, a statistical goodness-offit test is applied to evaluate significance of the difference between the empirical distribution and its theoretical counterpart at a predetermined level of significance. The need for a certain level of randomness to ensure unpredictability in the cryptography context makes procedures used to check cryptographic and classical randomness different from each other. The manipulations of random number sequences are required to make the cryptographic randomness tests more sensitive to small deviations from the exact randomness than their classical counterparts. The null hypothesis of the test is " $H_{0}$ : Sequences generated by the RNG of interest are random." There are more than a hundred alternative tests for the evaluation of cryptographic randomness available (L'Ecuyer and Hellekalek, 1998).

In the literature, some of these tests are grouped into test batteries or test suites (L'Ecuyer and Simard, 2007; Marsaglia and Tsang, 2002). A detailed review of test batteries is given by Demirhan and Bitirim (2016). To be qualified as suitable, an RNG should be identified as random in a predetermined portion or all of the tests in a test battery. The basic test battery is introduced by Knuth $(1998,1981$, 1969). Then, Marsaglia (1996) introduced the Diehard test battery composed of 12 randomness tests. Disadvantages of the Diehard test battery were overcame by another test battery called Dieharder that is introduced by Brown et al. (2014). Dieharder includes 26 cryptographic randomness tests. It is an improvement of the Diehard battery, provides a user friendly interface and a useful open source tool set for users of random numbers (Brown et al., 2014). The Dieharder test battery is implemented in the R package RDieHarder prepared by Eddelbuettel and Brown (2014). At the time of writing, Windows and OS X binaries are not available for this package. The US National Institute of Standards and Technology developed the NIST battery composed of 16 tests (Sýs et al., 2014; Sýs and Říha, 2014; Rukhin et al., 2010; Rukhin, 2001; Soto, 1999). The NIST battery is still used as a straightforward tool for formal certifications and accepted as a standard test battery. Sadique et al. (2012) reviewed the tests included in the NIST test battery. A suite of test batteries, TestU01, was introduced by L'Ecuyer and Simard $(2007,2014)$. TestU01 is a C library that combines most of the available randomness tests and RNGs in six test batteries (McCullough, 2006; L'Ecuyer and Simard, 2007). There are also smaller scale test batteries in terms of extensiveness. ENT was proposed by Walker (2014) that contains 5 statistics and tests. The Helsinki test battery is based on the Ising model and random walks on lattices and was proposed by Vattulainen et al. (1995). The Crypt-X test battery, which includes 6 tests, was developed by the Information Security Research Center at Queensland University of Technology (Sýs and Ríha, 2014; Soto, 1999). The SPRNG test battery includes some tests from the battery of Knuth (Mascagni and Srinivasan, 2000). Ruetti (2004) combined Knuth, Helsinki, Diehard, and SPRNG batteries and proposed a test battery consisting of 37 statistical and physical tests. 
In addition to the tests included in test batteries, there also exist recently proposed cryptographic randomness tests that are not performed by test batteries. Maurer (1992) proposed a statistical test for random bit generators. Hernandez et al. (2004) proposed a new test called Strict Avalanche Criterion (SAC). Ryabko et al. (2004) proposed an adaptation of the well-known chi-square test. This test is more efficient than the usual chi-square test in small samples. "Book Stack" and "Order" tests were proposed by Ryabko and Monarev (2005) for testing binary random bit sequences. Doganaksoy et al. (2006) proposed three randomness tests based on the random walk process. An advantage of these tests is that it is possible to calculate exact probabilities corresponding to the test statistics. The "Topological Binary Test" was introduced by Alcover et al. (2013) to test randomness in bit sequences. It counts different bit patterns of pre-determined length in a sequence of random bits.

Availability of a software implementing a test battery or even that of an individual cryptographic randomness test is a critical issue for the usefulness of the related test or battery. The library TestU01 is developed in ANSI C; hence, it is compiled by GNU tools instead of today's C compilers. Although TestU01 performs a wide variety of tests and their combinations, it lacks flexibility of implementation. Because the battery Dieharder is implemented in an R package, namely RDieHarder, it is more applicable and user-friendly than TestU01. However, unavailability of Windows and OS X binaries can be seen as a disadvantage that decreases its accessibility. A package for the implementation of the NIST battery is prepared for SUN workstations using ANSI C (Rukhin et al., 2010). Rukhin et al. (2010) provides a user guide for setting up the package and running the included tests. Ease of implementation of the NIST battery is similar with TestU01. For the implementation of individual randomness tests, there are also numerous $R$ packages such as randtests (Caeiro and Mateus, 2014) or DescTools (Signorell, 2015). Although some of the tests included in these packages are also used to evaluate cryptographic randomness, they cover neither recently proposed tests nor those developed specifically to test cryptographic randomness.

The usual chi-square test is applied with nearly all of the cryptographic randomness tests in the literature. The mentioned implementations including those covered by $\mathrm{R}$ automatically apply the chi-square test. However, there are numerous alternatives to the chi-square goodness-of-fit test such as the Kolmogorov-Smirnov, Anderson-Darling, or Jarque-Bera tests. It is apparent that because statistical qualities of these tests are better than the chi-square test, there will be a gain in performance of cryptographic randomness tests when applied with better goodness-of-fit tests. Thus, we need software that is capable of conducting actual cryptographic randomness tests such as topological binary, book stack, etc. with goodness-of-fit tests better than the usual chi-square test in statistical performance. When the range and variety of cryptographic randomness tests implemented by software and practicability of available software are considered, this software should effectively implement new tests with various goodness-of-fit tests and has a user-friendly interface. The package CryptRndTest (Demirhan, 2016) contributes to satisfy this need.

The aim of this article is to describe and illustrate the use of the R package CryptRndTest (currently in version 1.2.2) that performs some of recently proposed and basic cryptographic randomness tests. The package includes the functions adaptive . chi . square, bir thday. spacings, book. stack, GCD. test, topological.binary, and random.walk. tests to perform adaptive chi-square, birthday spacings, book stack, greatest common divisor, topological binary tests, and three tests based on the random walk process, respectively. To the best of our knowledge, the adaptive chi-square, topological binary, and the tests based on the random walk process are first implemented in software with package CryptRndTest. In addition to the chi-square procedure, these functions apply Anderson-Darling, Kolmogorov-Smirnov, and Jarque-Bera procedures when suitable. Because statistical performances of goodness-of-fit tests differ under various conditions, the application of different goodness-of-fit procedures is a beneficial feature. This is another important feature of package CryptRndTest. In addition, it has the following auxiliary functions: GCD, GCD. q, GCD . big, Strlng2, toBaseTwo, toBaseTen, and TBT. criticalvalue to compute the greatest common divisor under different conditions of inputs, approximately calculate the Stirling number of the second kind when the inputs are large, make base conversions precisely with large inputs, and calculate critical values for the topological binary test.

The paper is organized as follows: in the next section, methodologies of the tests included in package CryptRndTest are briefly given. Details of algorithms used to manipulate integer and bit sequences are mentioned, and applications of goodness-of-fit procedures performed by package CryptRndTest are clarified. Parameter settings and limitations for each test are mentioned. Finally, as an illustrative application of package CryptRndTest, random number generators available in $\mathrm{R}$ are tested by using the proposed package under different sequence and bit-length conditions. By this application, implementation performance of the package is analyzed, recently proposed tests are evaluated, and usage of package CryptRndTest is illustrated. 


\section{Performed tests}

\section{Adaptive chi-square}

The adaptive chi-square test was introduced by Ryabko et al. (2004). It is empirically demonstrated by Ryabko et al. (2004) that the adaptive chi-square test is more efficient than the classical chi-square test in the identification of non-random patterns in samples smaller than those required by the chi-square test. For example, when we work with 64-bit numbers the length of the alphabet is $2^{64}$; hence, we need to have a sequence of length greater than $5 \cdot 2^{64}$ to apply the classical chi-square test safely. The logic behind the test is to divide the alphabet into subsets and perform the chi-square test over subsets instead of individual elements of the sample. By this way, subsets are considered as a new alphabet and a new null hypothesis and its alternative are formed over the subsets. Because the number of categories required to test new hypotheses is equal to the number of subsets, the chi-square test is applied with much smaller samples. To conclude randomness, it is expected to observe a uniformity in the distribution of input numbers into the subsets. Deviations from this uniformity are detected by the adaptive chi-square test.

The function adaptive.chi.square() is called to apply the test. It implements the following pseudo-code algorithm:

\section{Algorithm 1.}

1. Input data as a matrix of bits or a vector of integers, the number of subsets $(S)$ that the alphabet will be divided into, and proportion of training data set;

2. If data is represented by bits, transform data to base-10;

3. Divide whole data set into training and testing subsets with regarding input weights;

4. Identify the numbers that are seen in the sequence of interest at least once;

5. Find the frequency of occurrences for each element of the alphabet in training and testing subsets;

6. For $i=1, \ldots, S$, find the frequency of elements that are seen $i$-times in the training and testing subsets;

7. Apply the two-sample chi-square test with the expected and observed counts obtained at the previous step over the training and testing subsets, respectively;

8. Return value of the test statistic, corresponding $p$-value, and the decision on the null hypothesis.

While working with integers, the alphabet corresponds to the range of considered numbers. For instance, if 32-bit numbers are being tested, the alphabet in Algorithm 1 includes the numbers between 0 and $2^{32}-1$. At step 4, we do not form the whole alphabet, instead we count the numbers (words) that are seen at least once; and hence, the rest of the numbers have zero count. At step 7, the degrees of freedom of the test is $S-1$.

Parameters of the adaptive chi-square test are: weight of training and testing samples $(r)$, the length of the considered number sequence $(n)$, and the number of subsets $(S)$ that the alphabet is divided into. Ryabko et al. (2004) do not give strict rules for the determination of values of these parameters. They suggest to run some experiments to find the values of parameters that provide the highest statistical performance such as power and specificity. Because such a study would not be cost-effective for an individual application of the test, at least, the user may evaluate sensitivity of test results to the values of $S$ and $r$. In the function adaptive. chi. square(), we set $r=0.5$ by default. The value of $S$ is set by the user. That of $n$ is determined by the length of input data. Because input data is a random sample from the RNG of interest, the value of $n$ should be increased with increasing bit-length to successfully represent the range of numbers that will be generated by the RNG. When bit-length is greater than 64, we utilize the package Rmpfr (Maechler, 2015) to work with higher precision.

Algorithm complexity of the function adaptive. chi . square () is $O\left(n^{2}\right)$ in the worst case. Required memory is directly related to the length of the input sequence. Due to the algorithm complexity of the function used to identify unique numbers at step 4, implementation time of the function adaptive.chi.square increases quadratically along with the length of the input sequence.

\section{Birthday spacings}

The Birthday Spacings test was given by Marsaglia and Tsang (2002). It focuses on the number of duplicated values of spacings between ordered birthdays among a year of pre-determined length. The observed duplication patterns in input numbers are compared with the patterns that should be observed under randomness. Thus, the birthday spacings test detects deviations from randomness 
by focusing on repetition frequency of numbers to ensure uniformity. Marsaglia and Tsang (2002) propose that the number of duplicated values is approximately distributed according to the Poisson distribution. They also derive an expression for the mean rate of the Poisson distribution.

The function birthday.spacings() is employed to run the test. It implements the following pseudo-code algorithm:

\section{Algorithm 2.}

1. Input data as a vector of integers of size $n$, the number of birthdays $(m)$, the length of year $(N)$, the mean rate of the theoretical Poisson distribution $(\lambda)$, and the number of classes $(k)$ that is constructed for goodness-of-fit tests;

2. Reshape the first $m \cdot\lfloor n / m\rfloor$ elements of input vector as a matrix of $\lfloor n / m\rfloor$ rows and $m$ columns;

3. Sort each row of the matrix of step 2 according to the values in columns;

4. For each row, find the distance between columns of the sorted matrix by extracting the values in the columns at the previous step;

5. Count duplicated values among the distances obtained at step 4;

6. Calculate class probabilities over the Poisson distribution with mean rate $\lambda$ for $x=0, \ldots, k$, and assign the rest of probability mass to the $(k+1)$-th class;

7. Calculate expected frequencies corresponding to the probabilities obtained at the previous step;

8. Replicate the expected counts to form the corresponding sample;

9. Apply the Anderson-Darling test to compare goodness-of-fit of the samples obtained at steps 5 and 8 ;

10. Apply the Kolmogorov-Smirnov test to compare goodness-of-fit of the samples obtained at steps 5 and 8;

11. Construct frequency table of the counts obtained at step 5;

12. Apply chi-square test over the frequency tables obtained at steps 7 and 11;

13. Return the values of test statistics, corresponding $p$-values, and decisions on the null hypothesis.

At step 2 of Algorithm 2, each row of the reshaped matrix includes birthdays in columns. Total number of rows determines the size of the sample that is used in goodness-of-fit tests applied at steps 9,10 , and 12. Manipulation of the input vector according to the birthday spacings test is completed at step 5. This manipulation produces the empirical sample in testing the goodness-of-fit to the Poisson distribution. The Anderson-Darling test at step 9 is applied by using function ad. test from the package kSamples (Scholz and Zhu, 2016). The Kolmogorov-Smirnov test at step 10 is applied by using function ks. test from the package stats.

Marsaglia and Tsang (2002) give some insight into the optimal values of parameters. The mean rate is $\lambda=m^{3} /(4 n)$. They state that for an RNG, it is harder to pass this test for increasing values of either $m$ or $n$. Specifically, the case with $m=4096$ and $n=2^{32}$ is qualified as a compelling setting for 32-bit generators. Length of the input sequence is another important parameter. Because the size of the sample used in testing the goodness-of-fit is equal to $\lfloor n / m\rfloor$, the length of the input sequence $(n)$ should be chosen large enough to apply the goodness-of-fit tests appropriately.

Algorithm complexity of the function birthday.spacings( $)$ is $O\left(n^{2}\right)$ in the worst case. The limitation of birthday. spacings () is directly related with the value of $m$. For all combinations of $m$ and $n$ suggested by Marsaglia and Tsang (2002), $\lambda$ is equal to 4 . Following this logic, when $n=2^{64}$ the value of $m$ giving $\lambda=4$ is $6,658,043$. In this case, for a reliable application of goodness-of-fit tests at steps 9,10 , and 12, we need at least 133,160,860 integers and correspondingly 8,522,295,040 bits. For bit lengths higher than 32 , the value of $\lambda$ can be taken as 2 . For instance, when $n=2^{64}$, the corresponding value of $m$ is $5,284,492$. Thus, decreasing the value of $\lambda$ does not overcome the need for a huge data set for a reliable testing. Note that use of huge data set for testing is a memory consuming operation.

\section{Book stack}

The Book Stack test was proposed by Ryabko and Monarev (2005). Positions of the numbers on a stack are taken into consideration. In this test, randomness implies that frequency of finding each number at each position is equally likely. Departures from this equality mean that some of the words are seen more frequently in contrast to the nature of randomness. The book stack test focuses on non-uniform patterns and frequent repetitions of input numbers to detect deviations from randomness by means of unexpected autocorrelation patterns and non-uniformity.

The function book. stack() implements the following pseudo-code algorithm to run the test: 


\section{Algorithm 3.}

1. Input data as a matrix of bits or a vector of integers and the number of subsets $(k)$ that the alphabet will be divided into;

2. If data are represented by bits, transform data to base-10;

3. Form an array that includes the numbers from 1 to the number of unique words in the input sequence;

4. Write each element of the input vector in place of the first element of the array formed at the previous step, and move the other elements except the one written to the first cell of the array one step right;

5. Record the array obtained at the previous step;

6. Go back to step 4 until all elements of the input vector are taken into account;

7. Divide the whole alphabet into $k$ non-overlapping subsets $\left(A_{1}, A_{2}, \ldots, A_{k}\right)$;

8. For each subset of the alphabet, find the frequency of occurrences of the number corresponding to the position of each element of input vector in the arrays formed at steps 4 and 5;

9. Apply the chi-square test with expected counts equal to $n \cdot A_{i}$, where $i=1, \ldots, k$ and $n$ is the length of input vector or number of columns of input matrix;

10. Return the value of test statistic, corresponding $p$-value, and decision on the null hypothesis.

In order to get an integer number of subsets, the length of input vector should be determined to get an integer as the length of subsets. Optimal value for the length of input vector is given as $n \approx B \cdot 2^{B / 2}$, where $B$ is the bit-length of the considered RNG (Ryabko and Monarev, 2005; Doroshenko and Ryabko, 2006; Doroshenko et al., 2006). For an appropriate determination of number of subsets, $k$, Ryabko and Monarev (2005) suggest performing an empirical study. As for an appropriate bit-length, it is mentioned by Ryabko and Monarev (2005) that it is hard to set up a sensible test with much higher bit-lengths.

Algorithm complexity of the function book. stack () is $O\left(n^{2}\right)$ in the worst case. The limitation of the Book Stack test is based on the bit-length of the considered RNG. For example, for $B=64$ the length of the input vector is calculated as $1.37 \cdot 10^{11}$ and we need 1 terabyte memory whereas the memory requirement is 4 megabytes for $B=32$. Due to both memory and sensibility issues, it is not appropriate to work with high bit-lengths such as 64 .

\section{Greatest common divisor}

Two tests proposed by Marsaglia and Tsang (2002) are based on the number of required iterations $(k)$ and the value of the greatest common divisor (GCD) obtained in the GCD operation. When perceived as random variables, both $k$ and GCD are independently and identically distributed and their distributions can be obtained under randomness. Marsaglia and Tsang (2002) derived distributions of $k$ with an empirical study and that of GCD theoretically under the null hypothesis of randomness. Departures from randomness imply nonconformity between empirical and theoretical distributions of $k$ and GCD. Thus, these tests focus on the deviations from independence and uniformity.

The function gcd.test() is called to apply the test. The following pseudo-code algorithm is implemented by gcd.test () when all of the goodness-of-fit tests are set to TRUE:

\section{Algorithm 4.}

1. Input data as an $N \times 2$ matrix of integers, mean and standard deviation of theoretical normal distribution of $k$;

2. Constitute a pair of numbers from each row of input matrix;

3. Apply the GCD operation to each pair formed at the previous step;

4. Store values of $k$ for $N$ pairs;

5. If the obtained GCD is less than 3, store it as 3 and if that of GCD is greater than 35, store it as 35;

6. Generate a random sample of size $N$ from the normal distribution with input values of mean and standard deviation.

7. If the tests based on $k$ will be conducted, go to the next step, otherwise go to step 13;

8. Apply the two sample Kolmogorov-Smirnov test in a two-sided setting to samples obtained at steps 4 and 6;

9. Apply the chi-square test to samples obtained at steps 4 and 6; 
10. Standardize the values of $k$ by using its empirical mean and standard deviation;

11. Apply the Jarque-Bera test to the standardized sample of step 10;

12. Apply the Anderson-Darling test to samples obtained at steps 4 and 6;

13. If the tests based on the GCD will be conducted, go to the next step, otherwise go to step 19;

14. Construct the cumulative distribution function (cdf) of the probability function (pf) of GCD given by Marsaglia and Tsang (2002);

15. Obtain theoretical frequencies for the GCD over the cdf of step 14. Specifically, if theoretical frequency of the GCD is less than 3, store it as 3 and if that of the GCD is greater than 35, store it as 35;

16. Replicate the expected counts to form the corresponding sample;

17. Apply the two sample Kolmogorov-Smirnov test in a two-sided setting to samples obtained at steps 5 and 16;

18. Apply the chi-square test to samples obtained at steps 5 and 16;

19. Return the values of calculated test statistics, corresponding $p$-values, and decisions on the null hypothesis.

Mean and standard deviation of the theoretical normal distribution for bit lengths other than 32 are not given by Marsaglia and Tsang (2002). We conducted extensive empirical studies, details of which are mentioned in the following sections, to obtain these parameters and tabulated obtained values in Table 3.

When bit-length is increased, corresponding value of GCD mostly becomes greater than 35; hence, the operation at step 15 of Algorithm 4 gets unreasonable. Thus, we observe that it is not appropriate to conduct tests based on the GCD for high bit-lengths such as 128 .

The Kolmogorov-Smirnov and chi-square tests at steps 8 and 17, and 9 and 18 are applied by using functions ks. test and chisq. test from the package stats, respectively. The Jarque-Bera test at step 11 is implemented by using the function jarque.bera. test from the package tseries (Trapletti and Hornik, 2015). The Anderson-Darling test is applied by using the function ad. test from the package kSamples.

Calculations of the number of required iterations and the value of the GCD are time consuming tasks for bit-lengths greater than 64 . To overcome this difficulty, we prepared three functions to calculate GCD-related variables. The first function GCD. q computes the number of required iterations, the value of the GCD, and the sequence of partial quotients by using the Euclidean algorithm. The function GCD is the recursive version of the Euclidean algorithm and it only provides the number of required iterations and the value of the GCD. The function GCD. big applies the Euclidean algorithm over multiple precision floating point numbers using package Rmpfr and provides all three outputs related with the GCD operation. While GCD is the fastest one, GCD. big gives the most precise results. It is also possible to use the binary GCD algorithm to decrease the implementation time. However, in this case it is not possible to apply tests over the number of required iterations of the Euclidean algorithm. When the GCD operation is done recursively, the algorithm complexity of gcd. test () is $O(\log (a))$, where $a$ is the maximum initial input to the recursive algorithm. Memory requirement for GCD tests is directly related with the value of $N$.

\section{Random walk tests}

In the literature, binary sequences are analyzed in detail by using the random walk process. Doganaksoy et al. (2006) proposed three tests based on the random walk stochastic process. In a random walk process, magnitude or direction of each change is determined by chance; hence, a random walk is random if increment and decrement probabilities are equal to each other. Therefore, random walk processes provide a good basis for randomness. In a random walk, a part of the sequence that intersects the $x$-axis with two successive points is called excursion, and over all excursions, the maximum distance from the $x$-axis is defined as height, and the vertical distance between minimum and maximum points over the $y$-axis is called expansion. Thus, we have three characteristics of the random walk process to observe deviations from randomness. The corresponding tests are called Random Walk Excursion, Random Walk Height, and Random Walk Expansion. If there is a trend in the process, the input sequence fails in the excursion test. The height test focuses on the moves with very low or high magnitude to detect non-randomness. The expansion test focuses on the anomalies in amplitude of the walk to identify non-random patterns. Because the exact probabilities corresponding to test statistics are calculated, the tests proposed by Doganaksoy et al. (2006) are also applicable for small sample sizes. 
The function random. walk. tests () is called to apply three tests, selectively. The following pseudocode algorithm is implemented by random. walk. tests() when all of the tests are to be applied:

\section{Algorithm 5.}

1. Input data as a matrix of bits of dimension $B \times k$, where $B$ is the bit length and $k$ is the length of input sequence;

2. Transform the input values from $\{0,1\}$ to $\{-1,1\}$;

3. To apply the expansion, excursion, and height tests go to steps 4,6 , and 7 , respectively;

4. For each non-overlapping set of length $B$, sum adjacent bits starting from the first bit and increasing by one at each iteration (By this way, we get $B$ summations for each number of interest);

5. For the Expansion test, count and store the summations of the previous step equal to zero;

6. For the Excursion test, calculate the maximum summation and the absolute value of the minimum summation among those of step 4 and store their sum;

7. For the Height test, store the absolute maximum of summations obtained at step 4;

8. Calculate theoretical cdf's and pf's for the tests regarding bit-lengths and probabilities tabulated by Doganaksoy et al. (2006);

9. Calculate empirical cdf's and pf's over the counts obtained at steps 5, 6, and 7;

10. Replicate the expected and empirical pf's to form the corresponding samples;

11. Apply the Anderson-Darling test to samples obtained at the previous step;

12. Apply the two sample Kolmogorov-Smirnov test in a two-sided setting to samples obtained at step 10;

13. Apply the chi-square test to samples obtained at step 10;

14. Return the values of calculated test statistics, corresponding $p$-values, and decisions on the null hypothesis.

The Anderson-Darling test at step 9 is applied by using function ad.test from the package kSamples. The Kolmogorov-Smirnov test at step 10 is applied by using function ks. test from the package stats. The chi-square test at step 11 is the classical application of the test without using a predefined function. If one of the tests is not applied, all the results related with that test in output are set to -1 .

Algorithm complexities of expansion, excursion, and height tests are $O(B), O(B\lfloor k \cdot B\rfloor)$, and $O(B\lfloor k \cdot B\rfloor)$, respectively. The limitation of the tests is unavailability of theoretical cdf's for bit-lengths other than $32,64,128$, and 256. Therefore, using the information given by Doganaksoy et al. (2006) the excursion is applied for bit-lengths of $16,32,64,128$, and 256; the height test is applied for bit-lengths of $64,128,256,512$, and 1024; and the expansion test is applied for bit-lengths of 32, 64, and 128. Although the size of required memory increases along with the length of the input sequence, it is possible to apply the tests with reasonable sequence lengths without causing memory pressure.

\section{Topological binary}

The topological binary test was proposed by Alcover et al. (2013) to test the randomness in bit sequences. The logic behind the test is based on the number of different fixed-length bit patterns in a bit sequence. Frequency of distinct non-overlapping bit patterns over the sequence of interest is influential on the test result. In case of randomness, we expect to have many different bit patterns in the input sequence. The main strength of the topological binary test is that it focuses on the number of bit patterns rather than frequency of occurrence of numbers. Because the exact distribution of the test statistic is derived, it is possible to apply the test for short bit sequences.

The function topological binary() implements the following pseudo-code algorithm to run the test:

\section{Algorithm 6.}

1. Input data as a $B \times k$ matrix of bits, where $B$ is the bit-length and $k$ is the length of considered number sequence, and the critical value;

2. Find and store non-overlapping blocks of length $B$;

3. Count the number of different $B$-bit patterns that appear across all the $k$ blocks; 


\begin{tabular}{|c|c|c|}
\hline & Function & Call \\
\hline Test & $\begin{array}{l}\text { GCD.test() } \\
\text { random.walk.tests() } \\
\text { birthday.spacings() } \\
\text { adaptive.chi.square() } \\
\text { book.stack() } \\
\text { topological.binary() }\end{array}$ & 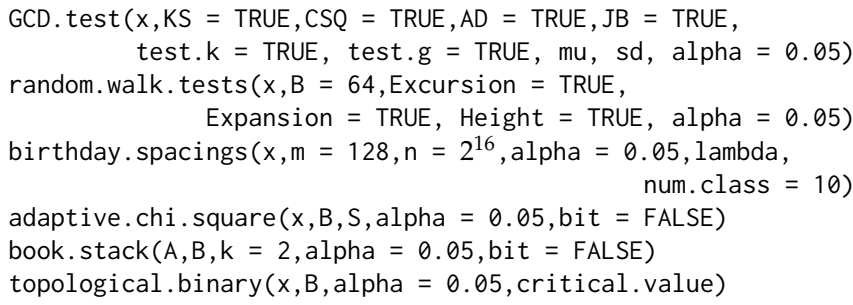 \\
\hline Auxiliary & $\begin{array}{l}\text { Strlng2() } \\
\text { GCD() } \\
\text { GCD.q() } \\
\text { GCD.big() } \\
\text { TBT.CriticalValue() } \\
\text { toBaseTen() } \\
\text { toBaseTwo() }\end{array}$ & $\begin{array}{l}\text { Strlng2 }(n, k, \log =\text { TRUE }) \\
\text { GCD }(x, y) \\
\text { GCD. } q(x, y) \\
\text { GCD. } \operatorname{big}(x, y, B) \\
\text { TBT.criticalValue }(m, k, a l p h a=0.01, \mathrm{cdf}=\text { FALSE, exact = TRUE) } \\
\text { toBaseTen }(x, m=128, \text { prec }=256, \text { toFile }=\text { FALSE, file }) \\
\text { toBaseTwo }(x, m=128, \text { prec }=512, \text { num. CPU }=4)\end{array}$ \\
\hline
\end{tabular}

Table 1: Usage of test and auxiliary functions of package CryptRndTest.

4. If the result of step 3 is less than one, then reject the null hypothesis;

5. Else if the result of step 3 is greater than $\min \left(k, 2^{B}\right)$, then do not reject the null hypothesis;

6. Else if the result of step 3 is less than the input critical value, then reject the null hypothesis;

7. Else do not reject the null hypothesis;

8. Return the result of step 3 as the value of test statistic and the decision on the null hypothesis.

Although the exact distribution of test statistic is derived by Alcover et al. (2013), calculation of the Stirling numbers of the second kind with large inputs is required with bit-lengths greater than 16 for the calculation of the cdf of the test statistics. Therefore, it is hard to obtain the critical value of the test for large bit-lengths by using available functions in R packages such as the function Stirling2 of package copula (Hofert et al., 2015). This case is a limitation of the function topological . binary(). To overcome this limitation of the test, we prepared the function TBT.CriticalValue to calculate required critical values for testing. Algorithm complexity of the function topological . binary() is $O\left(n^{2}\right)$ in the worst case. The required memory to run the topological binary test is related with the value of $k$.

\section{Auxiliary functions}

The package CryptRndTest has seven auxiliary functions, i.e., Strlng2(), $\operatorname{GCD}(), \operatorname{GCD} . q(), \operatorname{GCD} . \operatorname{big}()$, toBaseTwo(), toBaseTen(), and TBT.CriticalValue(). These functions are also suitable for individual use. Strlng2() is used to calculate critical values for the topological binary test implemented by TBT.Criticalvalue(). GCD() and GCD.q() are called to calculate the greatest common divisor in the GCD test implemented by gcd.test(). Three possible outcomes of the greatest common divisor operation are the number of iterations, the sequence of partial quotients, and the value of greatest common divisor. $G C D()$ provides all of these outcomes for any pair of integers excluding zero. Functions toBaseTwo() and toBaseTen() are used for base conversion from base 2 to 10 and vice versa for large integers.

The function Strlng2() is used to compute the natural logarithm of Stirling numbers of the second kind for large values of inputs in an approximate manner by the approaches of Bleick and Wang (1974) and Temme (1993). In this approach, Lambert W functions are employed at the log scale to overcome memory overflows.

Due to the large factorials in the calculation of Stirling numbers of the second kind, it is nearly impossible to compute the exact cdf of the topological binary test statistic for higher bit lengths without memory flows in R. The function TBT.CriticalValue() implements an approach for the calculation of the cdf and approximately computes the required critical value for the topological binary test at a given level of $\alpha$. Because TBT. CriticalValue() utilizes Strlng2(), accuracy of results decreases with increasing bit lengths and number of words under consideration. It is also possible to make exact calculations by TBT. CriticalValue(). In this case, the function Stirling2 from the package gmp (Lucas et al., 2014) is employed instead of Strlng2(). Because package gmp uses multiple precision arithmetic, implementation time of TBT.Criticalvalue() considerable increases. User should evaluate the trade off between implementation time and high precision.

Arguments of main and auxiliary functions of package CryptRndTest are summarized in Table 1. 


\begin{tabular}{cccc}
\hline & \multicolumn{3}{c}{ Sequence length } \\
\cline { 2 - 4 } Bit & Short (I) & Medium (II) & Long (III) \\
\hline 8 & 256 & 32768 & 65536 \\
16 & 16384 & 65536 & 131072 \\
32 & 32768 & 131072 & 262144 \\
64 & 131072 & 262144 & 524288 \\
128 & 131072 & 262144 & 524288 \\
\hline
\end{tabular}

Table 2: Lengths of random number sequences for different patterns.

\section{A numerical illustration}

As a numerical illustration of the package, we employed package CryptRndTest to test the randomness of RNG's available in R. By this way, we aim to get results of the tests that have not been applied to RNG's of interest yet, figure out implementation performance of package CryptRndTest under various scenarios, and illustrate some issues on the determination of parameters of the tests for considered scenarios. Note that it is impossible to observe the ability to control type-I error (rejection of randomness hypothesis while it is actually true) for the tests with an empirical study such as conducted in this section. Additionally, a more thorough investigation would be necessary to be able to reliably assess the algorithms, but this is out of scope of this article.

RNG's of interest are Wichmann-Hill (WH), Marsaglia-Multicarry (MM), Super-Duper (SD), Mersenne-Twister (MT), Knuth-TAOCP-2002 (KT02), Knuth-TAOCP (KT), and L'Ecuyer-CMRG (LE) (see the function Random in the base package for the details of these RNG's). Applied tests are topological binary (TBT), adaptive chi-square (Achi), birthday spacings (BDS), random walk expansion (RWT.Exp), random walk height (RWT.Hei), random walk excursion (RWT.Exc), book stack (BS), and greatest common divisor (GCD). TBT, RWT.Exp, RWT.Hei, and RWT.Exc tests work with binary numbers while the rest of the tests take integers as input. BDS and RWT tests are applied separately with each of Anderson-Darling, Kolmogorov-Smirnov, and chi-square goodness-of-fit tests, and the GCD test is applied separately with each of Anderson-Darling, Kolmogorov-Smirnov, Jargue-Bera, and chi-square goodness-of-fit tests. The total number of applied randomness tests is 21 . All the tests are applied at both 0.01 and 0.05 levels of significance and 8, 16, 32, 64, and 128-bit lengths. Considered lengths of random number sequences for each bit-length are given in Table 2.

Because we get unreasonable implementation times for longer sequences at the level of 128-bit, the same sequence lengths as 64-bit are considered for 128-bit numbers.

To conduct the adaptive chi-square test, we need to determine the value of argument $S$ and the proportions of training and testing samples. The latter one is taken equal. As for the value of $S$, we did not detect a significant change in the test results observed for medium sequence length for all bit-lengths for $S=2,3,4$ in pilot runs. The values greater than 4 increase the implementation time whereas small values decrease resolution. Thus, it is taken as 4 for all bit-lengths to work with a reasonable degrees of freedom in the chi-square test. Also, the adaptive chi-square test is applied for all bit-lengths.

Arguments of the birthday spacings test are the number of birthdays (m), the length of year (n), the mean rate of the theoretical Poisson distribution (lambda), and the number of classes (num.class), which is used for goodness-of-fit tests. In the experiments, the argument $\mathrm{m}$ was taken as 8,128 , and 4096 for 8,16 , and 32-bit-lengths, respectively. The argument $n$ was set to $2^{B}$, where $B$ is the bit-length. The argument lambda was calculated by the formula given by Marsaglia and Tsang (2002). The argument num. class was set to 5 and 10 for 8 and 16-bit and higher lengths, respectively.

For the book stack test, length of the sample (n) should be determined and data should be prepared according to the value of $\mathrm{n}$. Also, the number of subsets that the alphabet will be divided into $(\mathrm{k})$ should be determined. The formula proposed by Ryabko and Monarev (2005) is used to calculate the value of $\mathrm{n}$, and we set $\mathrm{k}=\mathrm{n} / B$.

In the GCD test procedure, tests are conducted for two outputs of the GCD operation, i.e., the number of iterations required to find GCD $(k)$ and GCD $(g)$ itself. The population distribution of $k$ is well approximated by a normal distribution and parameters of the normal distribution are given by Marsaglia and Tsang (2002) for 32-bit integers after an extensive numerical study. We observed that the parameters of the population distribution differ for different bit-lengths and conducted a numerical study to figure out the values of parameters for considered bit-lengths. For this study, $10^{6}$ 30-bit true random numbers were obtained from the web service "www.random.org." Then, they were converted to $8,16,32,64$, and 128-bit numbers. The GCD operation was applied and mean (mu.GCD) and standard deviation (sd.GCD) of $k$ were obtained as given in Table 3 after checking the normality of the empirical distribution by means of descriptive statistics and the Anderson-Darling goodness-of-fit 


\begin{tabular}{ccc}
\hline Bit & mu.GCD & sd.GCD \\
\hline 8 & 3.9991 & 1.6242 \\
16 & 8.8784 & 2.3664 \\
32 & 18.4023 & 3.4000 \\
64 & 31.3269 & 4.3349 \\
128 & 31.8390 & 4.3678 \\
\hline
\end{tabular}

Table 3: Mean and standard deviation of population distribution of $k$.

\begin{tabular}{ccccccc}
\hline & \multicolumn{3}{c}{$\alpha=0.01$} & & \multicolumn{3}{c}{$\alpha=0.05$} \\
\hline Bit & Short & Medium & Long & Short & Medium & Long \\
\hline 8 & 153 & NA & NA & 153 & NA & NA \\
16 & 14423 & 41268 & NA & 14423 & 41266 & NA \\
32 & 32767 & 131066 & 262129 & 32767 & 131066 & 262129 \\
64 & 131070 & 262113 & 523264 & 131070 & 262113 & 524264 \\
128 & 131072 & 262144 & 524288 & 131072 & 262144 & 524288 \\
\hline \multicolumn{7}{l}{ NA: not available. }
\end{tabular}

Table 4: Critical values for topological binary test.

test. The values obtained for 32-bit are very close to those obtained by Marsaglia and Tsang (2002).

As expected, the mean of $k$ increases along with bit-length, and it approaches 35 (Marsaglia and Tsang, 2002). The mild increase in the values of standard deviations is due to the increasing range of the numbers that can be generated with a given bit-length. Also, the GCD test is applied for all bit-lengths. However, nearly for all 128-bit random numbers, $g>35$. Due to the operation done at step 15 of Algorithm 4, it is unreasonable to conduct the GCD test over $g$ for 128-bit numbers.

The topological binary test is also applied for all bit-lengths. Critical values for the topological binary test are calculated by using the function TBT.criticalValue() for each bit and sequence length combination and presented in Table 4 . Because the length of sequence being tested cannot be longer than $2^{m}-1$, where $m$ is the bit-length, critical values for medium and long sequences at 8-bit and for long sequences at 16-bit levels are not available in Table 4.

In the application, random numbers were generated by using the same seed 283158301 . Let sim. data be an integer vector including data to be tested. It is reshaped with the following code according to bit-length $\mathrm{B}$ :

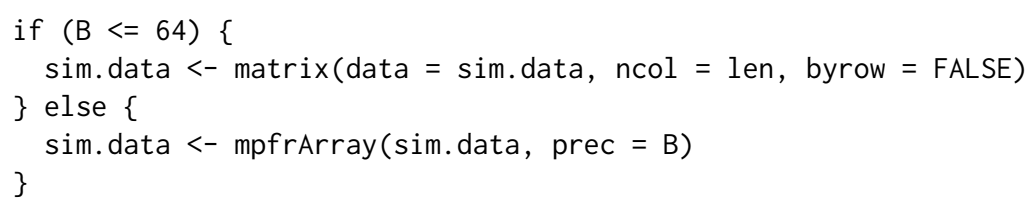

The adaptive chi-square, random walk, and topological binary tests were straightforwardly called with the mentioned arguments. For the book stack test, the following code is employed:

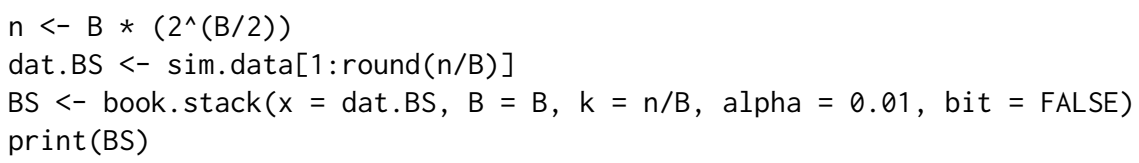

For the GCD tests, the input number sequence was divided into two-sequences and the tests were applied with the following code:

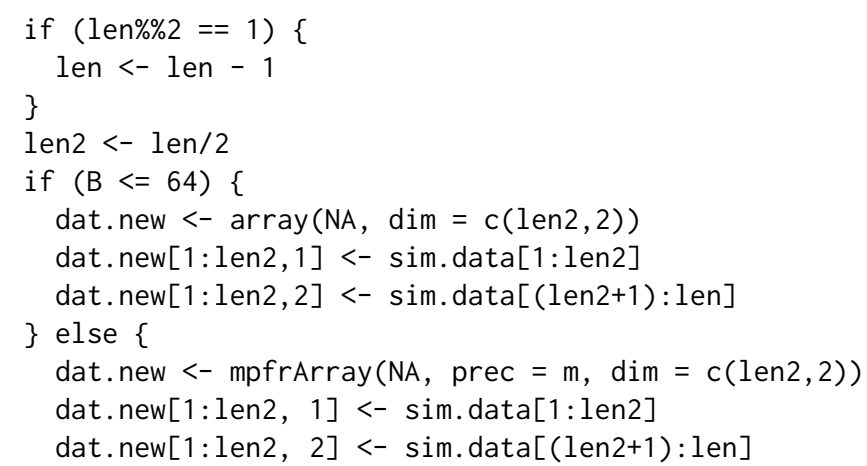




\begin{tabular}{cccccccccc}
\hline & & \multicolumn{1}{c}{ Tests } \\
\cline { 3 - 9 } Bit & Length & TBT & Achi & BDS & RWT.Exp & RWT.Hei & RWT.Exc & BS & GCD \\
\hline 8 & 32768 & 0.62 & 2.88 & 0.46 & NA & NA & NA & $<0.01$ & 1.31 \\
16 & 65536 & 1.70 & 5.70 & 0.46 & NA & NA & 4.33 & 0.02 & 3.74 \\
32 & 131072 & 6.68 & 10.88 & 2.10 & NA & 0.26 & 15.99 & 4253.01 & 12.32 \\
64 & 262144 & 32.05 & 86.31 & NA & 84.21 & 88.74 & 64.68 & NA & 37.36 \\
128 & 262144 & 77.16 & 10121.34 & NA & 221.16 & 196.96 & 149.29 & NA & 2657.62 \\
\hline
\end{tabular}

TBT: topological binary, Achi: adaptive chi-square, BDS: birthday spacings, RWT: random walk, Exp: expansion,

Hei: height, BS: book stack, GCD: greatest common divisor, Length: the length of random number sequence,

NA: not available.

Table 5: Mean implementation time for each test in seconds.

\}

$\mathrm{EBOB}<-\mathrm{GCD} \cdot \operatorname{test}(x=$ dat.new, $B=m, m u=m u . G C D, s d=$ sd.GCD, test.g = do.test.g) print (EBOB)

where len is the length of the input sequence. Whole code snippets used to implement experiments are given in the vignette of the package CrpytRndTest.

Random number sequences used for the performance analysis are of medium length given in Table 2 and generated by the WH generator under each bit level. Five replications were made for each test. Mean implementation times calculated over five replications are shown in Table 5 in seconds. All variances of implementation times are less than 0.01. BDS, RWT, and BS tests were not applied at all bit-lengths due to reasons explained in the relevant sections.

Implementation times of all tests from 8 to 64-bit levels are all sufficient. For 128 bits, most of the implementation times of Achi and GCD tests are taken by finding unique values in a sequence composed of multiple precision floating-point (mpf) numbers at step 4 of Algorithm 1 and the value of gcd for mpf numbers at step 3 of Algorithm 4, respectively. For these operations, mpf numbers are used via the package Rmpfr. The package Rmpfr is based on the GMP GNU library and provides an interface from $\mathrm{R}$ to $\mathrm{C}$ (Maechler, 2011, 2015). Due to the use of mpf numbers via the package Rmpfr, there is a considerable increase in implementation time of Achi and GCD tests at 128-bit level. However, the gain in precision is worth the delay in implementation of these tests. Performances of the tests working with binary numbers are all sufficient at the 128-bit level. Implementation time of the BS test exponentially increases along with the bit-length. Although it is reasonable for 32 bits, application of the test for higher bit-lengths requires an unreasonable amount of time for implementation.

All the tests were applied at both 0.01 and 0.05 levels of significance. The null hypothesis is " $H_{0}$ : Sequences generated by the RNG of interest are random" for all tests. For both levels of significance, success rates of RNGs over the total number of applied tests are given in Table 6. Detailed test results for the 0.05 level of significance are presented in the vignette of the package CrpytRndTest. The total number of applied tests is given in the last row of Table 6 for each test scenario. For example, because the birthday spacings test is not applied for 64 bit-length, the total number of applied tests is 17 for all sequence lengths. Note that the values given in Table 6 should not be confused with issues related with statistical performance of the tests such as type I error or power. Table 6 represents the proportion of RNG's that did not fail in the given number of tests. In addition, because each test is applied individually, the information presented by Table 6 should not be perceived as the results of the application of a test battery.

In general, the proportion of success decreases with increasing sequence and bit-lengths. According to the proportions of success, performance of the WH generator is satisfactory for 16 and 32-bit numbers for all sequence lengths. The reason of getting a decreasing success rate with increasing bit-length is that the random walk tests with all goodness-of-fit tests and the GCD test with the Jarque-Bera goodness-of-fit test reject the randomness hypothesis while the rest of the tests mostly accept the hypothesis for bit-lengths greater than 32. In detail, the WH generator successfully passes both of the TBT and Achi tests nearly in all bit-sequence length combinations. Results of AD and KS goodnessof-fit tests applied under both BDS and GCD tests (with $k$ ) are similar, and the CS test more likely decides randomness of the WH generator. It is unsuccessful in passing the random walk tests for high bit-lengths. The BS test concludes WH's randomness under all of the test conditions. The GCD with the JB goodness-of-fit test rejects the null hypothesis of randomness under all test conditions but the first one. At the 0.01 level of significance, there is nearly no change in the results. The WH generator passes the GCD test with CS goodness-of-fit test for $k$ at $(8, \mathrm{I}),(8, \mathrm{II})$ and $(32, \mathrm{I})$ scenarios, and the BDS test with the AD goodness-of-fit test at (16, II).

According to the proportions of success, the SD generator mostly passes the tests for 16 and 32-bit integers for all sequence lengths, and 8-bit integers for short and long sequences. Detailed test results 


\begin{tabular}{|c|c|c|c|c|c|c|c|c|c|c|c|c|c|c|c|c|}
\hline \multirow{4}{*}{$\begin{array}{c}\text { Level } \\
\text { of } \\
\text { Significance }\end{array}$} & \multirow[b]{4}{*}{ RNG } & \multicolumn{15}{|c|}{ Bit-length } \\
\hline & & \multicolumn{3}{|c|}{8} & \multicolumn{3}{|c|}{16} & \multicolumn{3}{|c|}{32} & \multicolumn{3}{|c|}{64} & \multicolumn{3}{|c|}{128} \\
\hline & & \multicolumn{15}{|c|}{ Sequence length } \\
\hline & & I & II & III & I & II & III & I & II & III & I & II & III & I & II & III \\
\hline \multirow[t]{7}{*}{0.01} & WH & 0.92 & 0.58 & 0.50 & 0.93 & 1.00 & 0.86 & 0.86 & 0.93 & 0.93 & 0.47 & 0.47 & 0.47 & 0.33 & 0.27 & 0.33 \\
\hline & SD & 0.92 & 0.58 & 0.75 & 0.93 & 0.93 & 0.93 & 0.93 & 0.93 & 0.93 & 0.41 & 0.47 & 0.35 & 0.33 & 0.33 & 0.27 \\
\hline & MT & 0.92 & 0.58 & 0.58 & 1.00 & 1.00 & 0.93 & 0.93 & 0.93 & 0.93 & 0.47 & 0.41 & 0.47 & 0.33 & 0.33 & 0.33 \\
\hline & MM & 0.92 & 0.58 & 0.75 & 0.93 & 0.93 & 1.00 & 1.00 & 0.93 & 0.93 & 0.47 & 0.41 & 0.35 & 0.33 & 0.33 & 0.27 \\
\hline & LE & 0.92 & 0.67 & 0.58 & 0.93 & 0.93 & 0.79 & 1.00 & 0.93 & 0.93 & 0.47 & 0.47 & 0.47 & 0.33 & 0.27 & 0.33 \\
\hline & KT & 0.92 & 0.67 & 0.58 & 0.93 & 0.93 & 0.93 & 1.00 & 0.93 & 0.93 & 0.41 & 0.47 & 0.47 & 0.27 & 0.33 & 0.33 \\
\hline & KT02 & 0.92 & 0.67 & 0.58 & 1.00 & 0.93 & 1.00 & 1.00 & 0.93 & 0.86 & 0.47 & 0.41 & 0.47 & 0.27 & 0.33 & 0.33 \\
\hline \multirow[t]{7}{*}{0.05} & WH & 0.83 & 0.50 & 0.50 & 0.93 & 0.93 & 0.86 & 0.79 & 0.93 & 0.93 & 0.47 & 0.47 & 0.47 & 0.33 & 0.27 & 0.33 \\
\hline & SD & 0.92 & 0.58 & 0.75 & 0.93 & 0.93 & 0.86 & 0.86 & 0.79 & 0.86 & 0.41 & 0.47 & 0.29 & 0.33 & 0.33 & 0.27 \\
\hline & MT & 0.67 & 0.50 & 0.58 & 0.93 & 0.86 & 0.86 & 0.93 & 0.86 & 0.93 & 0.41 & 0.41 & 0.47 & 0.33 & 0.33 & 0.33 \\
\hline & $\mathrm{MM}$ & 0.92 & 0.58 & 0.75 & 0.93 & 0.93 & 0.93 & 1.00 & 0.86 & 0.93 & 0.47 & 0.41 & 0.35 & 0.33 & 0.33 & 0.27 \\
\hline & LE & 0.92 & 0.67 & 0.58 & 0.93 & 0.93 & 0.79 & 0.93 & 0.86 & 0.93 & 0.47 & 0.41 & 0.47 & 0.33 & 0.27 & 0.33 \\
\hline & $\mathrm{KT}$ & 0.92 & 0.58 & 0.58 & 0.93 & 0.86 & 0.93 & 1.00 & 0.93 & 0.93 & 0.41 & 0.41 & 0.47 & 0.27 & 0.20 & 0.33 \\
\hline & KT02 & 0.83 & 0.58 & 0.42 & 1.00 & 0.93 & 0.93 & 1.00 & 0.93 & 0.79 & 0.47 & 0.41 & 0.47 & 0.27 & 0.33 & 0.33 \\
\hline \multicolumn{2}{|c|}{ Number of tests } & 12 & 12 & 12 & 15 & 15 & 15 & 15 & 15 & 15 & 17 & 17 & 17 & 15 & 15 & 15 \\
\hline
\end{tabular}

Table 6: Success rates for RNGs over the tests applied by package CryptRndTest.

for the SD generator at the 0.05 level of significance are similar to that of the WH generator for the TBT, Achi, BDS, RWT, and BS tests. It is better in the GCD test with the JB goodness-of-fit test for $k$. At the 0.01 level of significance, the CS goodness-of-fit test applied with the GCD test cannot reject the null hypothesis for 4 scenarios.

Reaction of the tests for MT, MM, and LE generators is similar to that of the WH generator. According to the proportions of success, success rates of the MT generator are satisfactory for 16 and 32-bit numbers for all sequence lengths; and that of the MM generator is very satisfactory for 16 and 32-bit numbers for all sequence lengths, and 8-bit numbers for short and long sequence lengths. Success proportions of LE, KT, and KT02 generators are high for 16 and 32-bit numbers for all sequence lengths, and 8-bit numbers for short sequences. The BS test rejects randomness of the KT02 generator for 8-bit numbers for all sequence lengths at the 0.05 level of significance. However, it cannot reject the null hypothesis for 8-bit numbers for all sequence lengths for $\alpha=0.01$.

For 64-bit numbers, only the random walk excursion test with AD and KS goodness-of-fit tests cannot reject the null hypothesis for all RNG's. None of the random walk tests decides randomness of RNG's for 128-bit numbers. RNG's pass TBT, Achi, and GCD for $k$ with AD, KS, and CS goodness-of-fit tests for almost all sequence lengths. This situation decreases the proportion of success for 64 and 128-bit numbers. This result would be due to the conservativeness of random walk height, random walk expansion tests, and GCD test with the Jarque-Bera goodness-of-fit test for higher bit lengths.

\section{Summary}

Statistical analysis of randomness of a cryptographic random number generator is a critical and necessary task to make use of the generator in cryptographic applications. Many cryptographic randomness tests are available for this task including recently proposed ones. Although there are several alternatives, the chi-square test is frequently employed within these cryptographic randomness tests as a goodness-of-fit test. In this regard, this article describes the package CryptRndTest that conducts frequently used and newly proposed 8 cryptographic randomness tests along with AndersonDarling, Kolmogorov-Smirnov, chi-square, and Jarque-Bera goodness-of-fit tests. Totally, package CryptRndTest runs 21 tests. It also provides auxiliary functions for the calculation of the greatest common divisor, sequence of partial quotients resulting from the greatest common divisor operation, the base conversion from 2 to 10 and vice versa, and the Stirling numbers of the second kind. All of these auxiliary functions also work with long integers by the use of multi-precision floating point numbers.

In addition to the description of package CryptRndTest, random number generators available in $\mathrm{R}$ are tested by 21 cryptographic randomness tests of CryptRndTest under various combinations of sequence and bit-lengths. Implementation performance of package CryptRndTest is also revealed by the numerical application.

The limitations of the package are mostly related to the memory and CPU capacities of the computer used to run functions of CryptRndTest. Because, increasing bit-length considerably decreases the implementation speed of tests working over integers, this can also be seen as a limitation for high bit-lengths. 


\section{Acknowledgments}

This work is fully supported by The Scientific and Technological Research Council of Turkey (TUBITAK) under Grant No. 114F249 of ARDEB-3001 programme. Authors wish to thank three anonymous reviewers and the editor for constructive comments that improved the quality and clarity of the article.

\section{Bibliography}

P. Alcover, A. Guillamon, and M. Ruiz. A new randomness test for bit sequences. Informatica, 24: 339-356, 2013. [p234, 239, 240]

W. Bleick and P. Wang. Asymptotics of Stirling numbers of the second kind. Proceedings of the American Mathematical Society, 42:575-580, 1974. [p240]

R. Brown, D. Eddelbuettel, and D. Bauer. Dieharder: A random number test suite (version 3.31.1). URL: http://www.phy.duke.edu/ rgb/General/dieharder.php, 2014. [Online; accessed 25-February2014]. [p233]

F. Caeiro and A. Mateus. randtests: Testing randomness in R. https: //CRAN. R-project. org/package= randtests, 2014. Online; accessed 2016-02-25. [p234]

H. Demirhan. CryptRndTest: Statistical Tests for Cryptographic Randomness, 2016. URL https: //CRAN. Rproject. org/package=CryptRndTest. R package version 1.2.1. [p234]

H. Demirhan and N. Bitirim. Statistical testing of cryptographic randomness. Journal of Statisticians: Statistics and Actuarial Sciences, 9:1-11, 2016. [p233]

A. Doganaksoy, C. Calik, F. Sulak, and M. Turan. New randomness tests using random walk. In Proceedings of National Cryptology Symposium II, Turkey, 2006. [p234, 238, 239]

S. Doroshenko and B. Ryabko. The experimental distinguishing attack on RC4. Cryptology ePrint Archive, Report 2006/070, 2006. http://eprint.iacr.org/. [p237]

S. Doroshenko, A. Fionov, A. Lubkin, V. Monarev, and B. Ryabko. On ZK-crypt, book stack, and statistical tests. Cryptology ePrint Archive, Report 2006/196, 2006. http://eprint.iacr.org/. [p237]

D. Eddelbuettel and R. Brown. RDieHarder: An R interface to the dieharder suite of random number generator tests. http://CRAN.R-project.org/package=RDieHarder, 2014. [Online; accessed 16June-2015]. [p233]

J. Hernandez, J. Sierra, and A. Seznec. The SAC test: A new randomness test, with some applications to PRNG analysis. In:Proceedings of the International Conference Computational Science and Its Applications, pages $960-967,2004$. [p234]

M. Hofert, I. Kojadinovic, M. Maechler, and J. Yan. copula: Multivariate Dependence with Copulas, 2015. URL http://CRAN. R-project.org/package=copula. R package version 0.999-14. [p240]

D. Knuth. The Art of Computer Programming, Volume 2 / Seminumerical Algorithms. Addison-Wesley, Reading, Massachusetts, 1 edition, 1969. [p233]

D. Knuth. The Art of Computer Programming, Volume 2 / Seminumerical Algorithms. Addison-Wesley, Reading, Massachusetts, 2 edition, 1981. [p233]

D. Knuth. The Art of Computer Programming, Volume 2 / Seminumerical Algorithms. Addison-Wesley, Reading, Massachusetts, 3 edition, 1998. [p233]

P. L'Ecuyer and P. Hellekalek. Random number generators: Selection criteria and testing. Random and Quasi-Random Point Sets Lecture Notes in Statistics, 138:223-265, 1998. [p233]

P. L'Ecuyer and R. Simard. TestU01: A C library for empirical testing of random number generators. ACM Transactions on Mathematical Software, 33:Article 22, 2007. [p233]

P. L'Ecuyer and R. Simard. TestU01: A C library for empirical testing of random number generators - user's guide, compact version. http://www.iro.umontreal.ca/ simardr/testu01/ guideshorttestu01.pdf, 2014. [Online; accessed 24-February-2014]. [p233]

A. Lucas, I. Scholz, R. Boehme, S. Jasson, and M. Maechler. gmp: Multiple Precision Arithmetic, 2014. URL https://CRAN. R-project. org/package=gmp. R package version 0.5-12. [p240] 
M. Maechler. Arbitrarily accurate computation with R: Package Rmpfr. https://CRAN. R-project. org/web/packages/Rmpfr/vignettes/Rmpfr-pkg. pdf, 2011. [Online; accessed 18-December-2015]. [p243]

M. Maechler. Rmpfr: R MPFR - Multiple Precision Floating-Point Reliable, 2015. URL https://CRAN. Rproject. org/package=Rmpfr. R package version 0.6-0. [p235, 243]

G. Marsaglia. Diehard: A battery of tests of randomness. http://stat.fsu. edu/ geo/diehard.html, 1996. [Online; accessed 25-February-2014]. [p233]

G. Marsaglia and W. Tsang. Some difficult to pass tests of randomness. Journal of Statistical Software, 7 (3):1-9, 2002. [p233, 235, 236, 237, 238, 241, 242]

M. Mascagni and A. Srinivasan. Algorithm 806: SPRNG: A scalable library for pseudorandom number generation. ACM Transactions on Mathematical Software, 26:436-461, 2000. [p233]

U. Maurer. A universal statistical test for random bit generators. Journal of Cryptology, 5:89-105, 1992. [p234]

B. McCullough. A review of TestU01. Journal of Applied Econometrics, 21:677-682, 2006. [p233]

M. Ruetti. A Random Number Generator Test Suite for the C++ Standard-Diploma Thesis. Institute for Theoretical Physics, ETH Zurich, 2004. [p233]

A. Rukhin. Testing randomness: A suite of statistical procedures. Theory of Probability and Its Applications, 45:111-132, 2001. [p233]

A. Rukhin, J. Soto, J. Nechvatal, M. Smid, E. Barker, S. Leigh, M. Levenson, M. Vangel, D. Banks, A. Heckert, J. Dray, and S. Vo. A statistical test suite for random and pseudorandom number generators for cryptographic applications. http://csrc.nist.gov/groups/ST/toolkit/rng/documents/ SP800-22rev1a.pdf, 2010. [Online; accessed 17-June-2015]. [p233, 234]

B. Ryabko and V. Monarev. Using information theory approach to randomness testing. Journal of Statistical Planning and Inference, 133:95-110, 2005. [p234, 236, 237, 241]

B. Ryabko, V. Stognienko, and Y. Shokin. A new test for randomness and its application to some cryptographic problems. Journal of Statistical Planning and Inference, 123:365-376, 2004. [p234, 235]

J. Sadique, U. Zaman, and R. Ghosh. Review on fifteen statistical tests proposed by NIST. Journal of Theoretical Physics and Cryptography, 1:18-31, November 2012. [p233]

F. Scholz and A. Zhu. kSamples: K-Sample Rank Tests and Their Combinations, 2016. URL https: //CRAN. Rproject. org/package=kSamples. R package version 1.2-3. [p236]

A. Signorell. DescTools: Tools for descriptive statistics. https://CRAN.R-project.org/package= DescTools, 2015. Online; accessed 2016-02-25. [p234]

J. Soto. Statistical testing of random number generators. In Proceedings of the 22nd National Information Systems Security Conference, USA, 1999. National Institute of Standards and Technology. [p233]

M. Sýs and Z. Říha. Faster randomness testing with the NIST statistical test suite. In R. Chakraborty et al., editors, Security, Privacy, and Applied Cryptography Engineering Lecture Notes in Computer Science, pages 272-284, Portugal, 2014. Springer. [p233]

M. Sýs, P. Švenda, M. Ukrop, and V. Matyáš. Constructing empirical tests of randomness. In A. H. Mohammad S. Obaidat and P. Samarati, editors, SECRYPT 2014 Proceedings of the 11th International Conference on Security and Cryptography, pages 229-237, Portugal, 2014. SCITEPRESS - Science and Technology Publications. [p233]

N. Temme. Asymptotic estimates of Stirling numbers. Studies in Applied Mathematics, 89:233-243, 1993. [p240]

A. Trapletti and K. Hornik. tseries: Time Series Analysis and Computational Finance, 2015. URL http: //CRAN.R-project. org/package=tseries. R package version 0.10-34. [p238]

I. Vattulainen, T. Ala-Nissila, and K. Kankaala. Physical models as tests of randomness. Physical Review Engineering, 52:3205-3213, 1995. [p233]

J. Walker. ENT - A pseudorandom number sequence test program. https://www. fourmilab.ch/ random/, 2014. [Online; accessed 25-February-2014]. [p233] 


\author{
Haydar Demirhan \\ Hacettepe University \\ Department of Statistics 06800 Beytepe Ankara \\ Turkey \\ and \\ RMIT University \\ School of Science \\ Mathematical Sciences \\ 3001 Melbourne Victoria \\ Australia \\ haydarde@hacettepe.edu. tr \\ haydar.demirhan@rmit.edu.au \\ Nihan Bitirim \\ Council of Higher Education \\ 06800 Cankaya Ankara \\ Turkey
}

